\title{
Decreased expression of long non-coding RNA GAS5 promotes cell proliferation, migration and invasion, and indicates a poor prognosis in ovarian cancer
}

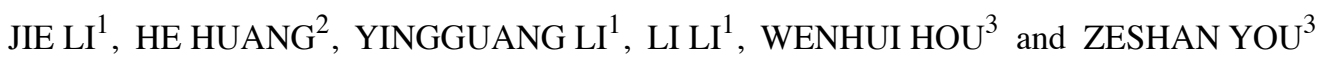 \\ ${ }^{1}$ Department of Gynecology, The Eastern Hospital of the First Affiliated Hospital of Sun Yat-sen University, Guangzhou, \\ Guangdong 510700; ${ }^{2}$ Department of Gynecology, Sun Yat-sen University Cancer Center, Guangzhou, Guangdong 510000; \\ ${ }^{3}$ Department of Gynecology, The First Affiliated Hospital of Sun Yat-sen University, Guangzhou, Guangdong 510080, P.R. China
}

Received April 11, 2016; Accepted May 27, 2016

DOI: $10.3892 /$ or.2016.5200

\begin{abstract}
Long non-coding RNA growth arrest-specific 5 (GAS5) was reported to be aberrantly expressed in various types of cancers. However, the role of GAS5 in the evolution and progression of ovarian cancer remains elusive. In the present study, we aimed to investigate the cellular function and clinical significance of GAS5 in ovarian cancer. GAS5 expression levels in 63 ovarian cancer tissues were detected by quantitative real-time PCR. Cell Counting Kit-8 (CCK-8) assay was performed to analyze the effect of GAS5 on cell proliferation. The effect of GAS5 on cell migration and invasion was detected using Transwell assay. Cell apoptosis was evaluated by flow cytometry and Hoechst staining. SKOV3 cells with stable expression of GAS5 were injected into nude mice to study the effect of GAS5 on tumorigenesis in vivo. Western blotting was used to determine the protein levels of GAS5 potential targets. The results showed that GAS5 was markedly decreased in tumor tissues and a lower expression of GAS5 was detected in tumors with larger size, deeper invasive depth and higher tumor stage. Patients with low GAS5 expression level had poorer disease-free $(\mathrm{P}<0.0001)$ and overall survival $(\mathrm{P}=0.0016)$ than those with high GAS5 expression. Moreover, overexpression of GAS5 was demonstrated to suppress ovarian cancer cell proliferation in vitro and in vivo. Finally, we found that GAS5 influenced ovarian cancer cell proliferation, partly via regulating cyclin D1, p21 and apoptosis protease activating factor 1 (APAF1) expression. Our findings suggest that lncRNA GAS5 may represent a novel indicator of poor prognosis in
\end{abstract}

Correspondence to: Dr Zeshan You, Department of Gynecology, The First Affiliated Hospital of Sun Yat-sen University, 58 Zhongshan Road II, Guangzhou, Guangdong 510080, P.R. China E-mail: youzeshan888@21cn.com

Key words: ovarian cancer, long non-coding RNA, growth arrest-specific 5, poor prognosis, cell proliferation, apoptosis ovarian cancer and may be a potential therapeutic target for diagnosis and therapy.

\section{Introduction}

Ovarian cancer is one of the most lethal gynecologic malignancy, which accounts for $3 \%$ of cancers among women, and the fifth leading cause of death among the female population (1). In spite of the development in cancer therapy, ovarian cancer mortality has virtually remained unimproved for the last two decades (1). Thus, early diagnosis and estimation of the risk of future progression have a significant impact on the prognosis of ovarian tumors. In order to improve the long-term survival of women with ovarian cancer, intensive research efforts have been made toward the detection and treatment of ovarian cancer for the last 10 years, but with only modest improvement (2). Therefore, a better understanding of the pathogenesis and identification of the molecular alterations is urgently required for the development of useful indicators that aid novel effective therapies for ovarian cancer.

It is well known that less than $2 \%$ of the genome serves as the transcription template for protein-coding sequences, whereas up to $70 \%$ of the human genome can be transcripted into non-coding RNAs (3). Most of the non-coding transcripts are termed non-coding RNAs (ncRNAs). The non-coding RNAs are classified as small or long ncRNAs (lncRNAs). lncRNAs are a group of RNAs that are more than $200 \mathrm{nt}$ in length with limited or no protein-coding capacity (4,5). Most lncRNAs are evolutionarily conserved and their expression is strictly regulated, playing critical roles in the regulation of gene expression (6). IncRNAs often display dysregulated expression and are involved in the initiation and progression of human cancers $(7,8)$. A large body of evidence has revealed the contribution of lncRNAs as having oncogenic and tumor-suppressor roles in tumorigenesis. IncRNA Hox transcript antisense intergenic RNA (HOTAIR) is well known as a famous oncogenic lncRNA involved in tumor pathogenesis. IncRNA HOTAIR has been consistently upregulated and identified as a strong prognostic biomarker of patient outcomes such as metastasis and patient survival in different human cancers (9-11). IncRNA metastasis-associated lung 
adenocarcinoma transcript 1 ( MALAT1) plays a role as an oncogene, which was found to be upregulated in several types of cancer tissues and it controls cell proliferation and cell cycle progression $(12,13)$. lncRNA antisense non-coding RNA in the INK4 locus (ANRIL) is a well-known tumor-suppressor gene, which interacts with the PRC2 complex to block the activity of $\mathrm{p} 15^{\mathrm{INK} 4 \mathrm{~B}}(14)$. Furthermore, ANRIL plays a role as an oncogene in bladder cancer and regulates bladder cancer cell proliferation and apoptosis through the intrinsic apoptosis pathway (15). lncRNA maternally expressed gene 3 (MEG3) also plays a role as a tumor-suppressor gene, which is downregulated in various primary human cancers. Overexpression of MEG3 was found to induce cell growth and promote cell apoptosis $(16,17)$. Nevertheless, the overall pathophysiological contributions of lncRNAs to ovarian cancer remain unknown.

In the present study, we aimed to determine the clinical significance and functions of dysregulated lncRNAs in ovarian carcinogenesis. IncRNA growth arrest-specific transcript 5 (GAS5) was previously found to be consistently downregulated and identified as a tumor suppressor in stomach cancer, prostate and breast cancer cells, and gastric cancer (18-21), although its functional significance has not yet been established. However, the role of 1ncRNA GAS5 in ovarian cancer remains unknown. In the present study, we demonstrated that decreased expression of GAS5 was a characteristic molecular change in ovarian cancer and analyzed the potential relationship between the GAS5 expression level in tumor tissues and clinicopathological features of ovarian cancer. Furthermore, we also investigated the effect of altered GAS5 on the phenotypes of ovarian cancer cells in vitro and in vivo. Our findings suggest that lncRNA GAS5 may represent a novel indicator of poor prognosis in ovarian cancer and may be a potential therapeutic target for diagnosis and therapy.

\section{Materials and methods}

Tissue collection. Between 2008 and 2010, 63 ovarian cancer tissues and matched adjacent normal tissues were obtained from patients who underwent surgery at the First Affiliated Hospital of Sun Yat-sen University, and were diagnosed with ovarian cancer (stages II, III and IV) based on histopathological evaluation according to the International Federation of Obstetrics and Gynecology (FIGO) criteria. Clinical pathology information was available for all samples (Table I). No local or systemic treatment was conducted in these patients before the operation. All tissues were collected and immediately frozen in liquid nitrogen and stored at $-80^{\circ} \mathrm{C}$ until RNA extraction. The present study was approved by the Research Ethics Committee of the First Affiliated Hospital of Sun Yat-sen University. Informed consent was obtained from every participant.

Cell lines and culture conditions. Human ovarian surface epithelial cells (IOSE25) were immortalized and cultured as previously described (22). Five human ovarian cancer cell lines, A2780, OVCAR3, SKOV3 and 3AO, were purchased from the American Type Culture Collection (ATCC) (Manassas, VA, USA) and the Cell Bank of the Chinese Academy of Sciences
(Shanghai, China), respectively. The ovarian cancer cell lines were maintained according to the vendor's instructions. In brief, A2780 and 3AO cell lines were routinely cultured in Dulbecco's modified Eagle's medium (DMEM) with $10 \%$ fetal bovine serum (FBS) (Gibco, Carlsbad, CA, USA). SKOV3 cells were cultured in McCoy's 5A Medium Modified (ATCC) with $10 \%$ FBS (Gibco). OVCAR3 cells were cultured in RPMI1640 medium (ATCC) with 20\% FBS (Gibco). All the media contained $1 \%$ penicillin-streptomycin $(100 \mathrm{U} / \mathrm{ml}$ penicillin and $100 \mu \mathrm{g} / \mathrm{ml}$ streptomycin). All ovarian cancer cells were cultured and maintained in a humidified incubator at $37^{\circ} \mathrm{C}$ and supplemented with $5 \% \mathrm{CO}_{2}$.

RNA extraction and quantitative real-time PCR (qRT-PCR) analysis. Total RNA was extracted from tissue samples or cultured cells using TRIzol reagent (Invitrogen Inc., Carlsbad, CA, USA). For qRT-PCR, $2 \mu \mathrm{g}$ of total RNA was used for reverse transcription reaction and cDNA synthesis using a Reverse Transcription kit (Takara, Dalian, China). Quantitative real-time PCR analyses were performed using SYBR-Green Real-Time Master Mix (Toyobo, Tokyo, Japan). Results were normalized to a constitutive expression gene, GAPDH. The PCR primers for GAS5 were: 5'-CTTCTGGGCTCAAGTG ATCCT-3' (forward) and 5'-TTGTGCCATGAGACTCCA TCAG-3' (reverse); for GAPDH, 5'-ACACCCACTCCTCCAC CTTT-3' (forward) and 5'-TTACTCCTTGGAGGCCATGT-3' (reverse). qRT-PCR and data collection were performed on Applied Biosystems 7500 Sequence Detection system (ABI, USA). The relative expression of GAS5 was calculated and normalized using the $2^{-\Delta \Delta \mathrm{Ct}}$ method relative to GAPDH.

Protein extraction and western blotting. Total proteins were extracted from tissue samples or cultured cells with SDS lysis buffer (Beyotime, Shanghai, China) on ice for $20 \mathrm{~min}$ and the protein concentration was determined using BCA Protein Assay kit (Pierce, Rockford, IL, USA). Equal amounts of total proteins were separated by $10 \%$ SDS-polyacrylamide gel electrophoresis (SDS-PAGE), transferred to $0.22 \mu \mathrm{m}$ polyvinylidene difluoride (PVDF) membranes (Millipore, Billerica, MA, USA) and incubated with specific antibodies. Proteins were detected by enhanced chemiluminescence (ECL) as described by the manufacturer (Beyotime) and the intensity of the bands was quantified by densitometry (Quantity One software; Bio-Rad, Hercules, CA, USA). GAPDH antibody (1:2,000; Abcam) was used as control, anti-cyclin D1 (1:800), P21 $(1: 1,500)$ and apoptosis protease activating factor 1 (APAF1) $(1: 1,000)$ were purchased from Abcam.

Plasmid construction. To generate a GAS5 expression vector, the entire sequence of human GAS5 (2,651 bp) was synthesized and subcloned into the pCDNA4.1 vector. In addition, the plasmids were confirmed by DNA sequencing (Sangon, Shanghai, China).

Cell transfection. Ovarian cancer cells were seeded in 24-well plates $\left(1 \times 10^{5}\right.$ cells/well $)$ and incubated for $24 \mathrm{~h}$, and then the cells were transfected with pCDNA3.1-GAS5 $(2 \mu \mathrm{g})$ or the empty vector $(2 \mu \mathrm{g})$ using Lipofectamine 2000 (Invitrogen) in serum-free medium in accordance with the manufacturer's instructions. 
Cell proliferation assays. The cell proliferation assay was performed with Cell Counting Kit-8 (CCK-8) (Dojindo, Kumamoto, Japan) according to the manufacturer's instructions. In brief, $2 \times 10^{4}$ cells/well were seeded in a 96-well plate. Cell viability was evaluated with CCK- 8 at daily intervals from the next 24,48 and $72 \mathrm{~h}$ after seeding. After treatment with CCK- 8 at $37^{\circ} \mathrm{C}$ for $1 \mathrm{~h}$, ovarian cancer cells were used to measure the absorbance at $450 \mathrm{~nm}$ using a microplate reader Thermo Plate (Rayto Life and Analytical Sciences Co., Ltd., Germany).

Cell migration and invasion assays. Twenty-four-well Transwell inserts with $8-\mu \mathrm{m}$ pore size (Corning Costar, Inc., Corning, NY, USA) were used for Transwell migration and Matrigel invasion assays, separately. For the Transwell migration assay, $2 \times 10^{4}$ ovarian cancer cells suspended in $100 \mu \mathrm{l}$ serum-free corresponding culture medium were loaded into the upper chamber of the Transwell insert with a non-coated membrane. For the Transwell invasion assay, $4 \times 10^{4}$ ovarian cancer cells were plated in $100 \mu \mathrm{l}$ corresponding culture medium without FBS in the upper Matrigel-coated chamber instead. In both assays, $500 \mu 1$ of culture medium containing $20 \%$ FBS was added to the lower chamber for culture. Cells were then allowed to migrate or invade for $24 \mathrm{~h}$ at $37^{\circ} \mathrm{C}$. The cells that migrated or invaded into the bottom chamber were fixed with $100 \%$ methanol for 30 min and stained using $0.5 \%$ crystal violet (Sigma, St. Louis, MO, USA) for $20 \mathrm{~min}$, and the permeating cells were counted under a phase-contrast microscope (Olympus, Tokyo, Japan). All experiments were independently repeated at least three times.

Cell apoptosis assay. Cell apoptosis was evaluated by flow cytometry using an Annexin V-FITC/propidium iodide (PI) apoptosis detection kit (BestBio, Shanghai, China) in accordance with the manufacturer's instructions. Briefly, cells were harvested and washed with cold phosphate-buffered saline (PBS). Cells $\left(1 \times 10^{6}\right)$ were resuspended and stained using the Annexin V-FITC/PI apoptosis detection kit according to the manufacturer's instructions. Then, the samples were analyzed using Becton-Dickinson flow cytometer. Annexin V(+)/PI(-) and Annexin $\mathrm{V}(+) / \mathrm{PI}(+)$ represent the ovarian cancer cells in early and late apoptosis/necrosis, respectively. Moreover, cell apoptosis was also detected using Hoechst staining. Ovarian cancer cells were cultured in 6-well cell culture plates, and Hoechst 33342 (Sigma) was added to the culture medium. Fluorescence microscopy with a filter for Hoechst 33342 $(365 \mathrm{~nm})$ was used to detect nuclear morphology.

Tumor formation assay in a nude mouse model. Four-week-old female BALB/c athymic nude mice (Vital River Laboratory Animals, Beijing, China) were maintained under specific pathogen-free conditions and manipulated according to protocols approved by the Ethics Committee of Animal Experiments of the First Affiliated Hospital of Sun Yat-sen University. pCDNA3.1-GAS5 or pcDNA3.1 vector stably transfected SKOV3 cells were harvested and washed with PBS, then resuspended at a concentration of $5 \times 10^{7}$ cells $/ \mathrm{ml}$. A volume of $100 \mu \mathrm{l}$ of the suspended cells was subcutaneously injected into the right side of the posterior flank of each mouse. The subcutaneous tumor growth was examined every seven days, and tumor volumes were calculated using the equation: $\mathrm{V}=0.5 \times \mathrm{D} \times \mathrm{d}^{2}$ (V, volume; $\mathrm{D}$, longitudinal diameter; $\mathrm{d}$, latitudinal diameter). At five weeks post injection, the mice were sacrificed and tumor weights were measured. Each neoplasm was collected and stored at $-80^{\circ} \mathrm{C}$ for further analysis. All procedures performed in these studies involving animals were in accordance with the ethical standards of the Guide for the Care and Use of Laboratory Animals of the National Institutes of Health.

Statistical analysis. For quantitative data, all results are expressed as the mean \pm SD. Statistical analysis was performed using SPSS 18.0 (SPSS, Inc., Chicago, IL, USA). Statistical significance between groups was determined using a Student's t-test or a Chi-square test. Survival analysis was performed using the Kaplan-Meier method, and the log-rank test was used to compare the differences between patient groups.

\section{Results}

GAS5 is downregulated in human ovarian cancer tissues. To investigate the expression level of GAS5 in human ovarian cancer tissues, qRT-PCR was used to examine GAS5 expression in 63 paired ovarian cancer samples and matched adjacent normal tissues. As shown in Fig. 1A, the expression level of GAS5 was significantly downregulated in the ovarian cancer tissues compared with that in the corresponding adjacent histological normal tissues. In tumor specimens, the expression level of GAS5 was lower than that of the normal tissues, with the median ratio of 0.69 compared with the normal counterparts.

\section{Relationship between GAS5 expression and clinicopatho-} logical factors of the ovarian cancer patients. The clinical pathological findings of 63 ovarian cancer patients are listed in Table I. The 63 ovarian cancer patients were classified into two groups based on the median ratio of relative GAS5 expression (0.69) in the tumor tissues: group 1, high GAS5 expression group ( $n=32$, GAS5 expression ratio $\geq$ median ratio); group 2, low GAS5 expression group ( $\mathrm{n}=31$, GAS5 expression ratio $\leq$ median ratio) (Fig. 1B). The clinicopathological factors compared between the two groups are shown in Table II. The low-GAS5 group (group 2) was correlated with larger tumor size $(\mathrm{p}=0.0024)$, deeper depth of invasion $(\mathrm{P}=0.0009)$ and higher TNM stage $(\mathrm{P}=0.0379)$ than the high-GAS5 group (group 1). However, the GAS5 expression level was not associated with other parameters such as age $(\mathrm{P}=0.4527)$, histologic differentiation $(\mathrm{P}=0.8347)$ and lymphatic metastasis $(\mathrm{P}=0.5321)$ (Table II).

Association of GAS5 expression with patient survival. In order to confirm whether the GAS5 expression level is correlated with the outcome of ovarian cancer patients after ovariectomy, disease-free survival (DFS) and overall survival (OS) curves were plotted according to GAS5 expression level by the Kaplan-Meier analysis and log-rank test, respectively, and the results are presented in Fig. 1C and D. Markedly, patients with a low GAS5 expression level had poorer disease-free survival $(\mathrm{P}<0.0001)$ and overall survival $(\mathrm{P}=0.0016)$. For OS, the 3 -year overall accumulative survival rates of ovarian cancer 
Table I. Clinicopathological characteristics and GAS5 expression in 63 patient samples of ovarian cancer.

\begin{tabular}{|c|c|}
\hline Clinical parameters & No. of cases $(\%)$ \\
\hline \multicolumn{2}{|l|}{ Age (years) } \\
\hline$\leq 55$ & $29(46.03)$ \\
\hline$>55$ & $34(53.96)$ \\
\hline \multicolumn{2}{|l|}{ Tumor size (cm) } \\
\hline$\geq 5$ & $33(52.38)$ \\
\hline$<5$ & $30(47.62)$ \\
\hline \multicolumn{2}{|c|}{ Histologic differentiation } \\
\hline Well & $5(7.94)$ \\
\hline Moderate & $23(36.51)$ \\
\hline Poor & $28(44.44)$ \\
\hline Undifferentiated & $7(11.11)$ \\
\hline \multicolumn{2}{|l|}{ Invasive depth } \\
\hline $\mathrm{T} 1$ & $15(23.81)$ \\
\hline $\mathrm{T} 2$ & $16(25.40)$ \\
\hline $\mathrm{T} 3$ & $18(28.57)$ \\
\hline $\mathrm{T} 4$ & $14(22.22)$ \\
\hline \multicolumn{2}{|l|}{ FIGO stage } \\
\hline I & $8(12.70)$ \\
\hline II & $22(34.92)$ \\
\hline III & $27(42.86)$ \\
\hline IV & $6(9.52)$ \\
\hline \multicolumn{2}{|l|}{ Lymphatic metastasis } \\
\hline Yes & $30(47.62)$ \\
\hline No & $33(52.38)$ \\
\hline \multicolumn{2}{|l|}{ Distant metastasis } \\
\hline Yes & $8(12.70)$ \\
\hline No & $55(87.30)$ \\
\hline \multicolumn{2}{|l|}{ Expression of GAS5 } \\
\hline Low expression & $31(50.79)$ \\
\hline High expression & $32(49.21)$ \\
\hline
\end{tabular}

GAS5, growth arrest-specific 5; FIGO, International Federation of Obstetrics and Gynecology.

patients with a high level and a low level of GAS5 expression were 47.68 and $22.36 \%$, respectively. Low GAS5 expression indicated a shorter overall survival time for the ovarian cancer patients (median OS, 13 months) compared with high GAS5 expression patients (median OS, 29 months). Moreover, the 3-year disease-free survival rates for high GAS5 expression and low GAS5 expression were 36.59 and $19.76 \%$, respectively. The median survival time of patients with high GAS5 expression was 23 months, while the value was 6 months for patients with low GAS5 expression.

Overexpression of GSA5 suppresses ovarian cancer cell proliferation in vitro. To evaluate the impact of GAS5 on ovarian cancer cell proliferation, the expression level of GAS5 in a variety of cell lines, including ovarian cancer cell
Table II. Relationship between GAS5 expression and clinicopathological factors of the ovarian cancer patients.

\begin{tabular}{|c|c|c|c|}
\hline \multirow[b]{2}{*}{ Clinical parameters } & \multicolumn{2}{|c|}{ GAS5 } & \multirow[b]{2}{*}{ P-value } \\
\hline & Group 1 & Group 2 & \\
\hline Age (years) & & & 0.4527 \\
\hline$\leq 55$ & 16 & 13 & \\
\hline$>55$ & 15 & 19 & \\
\hline Tumor size $(\mathrm{cm})$ & & & 0.0024 \\
\hline$\geq 5$ & 10 & 23 & \\
\hline$<5$ & 22 & 8 & \\
\hline $\begin{array}{l}\text { Histologic } \\
\text { differentiation }\end{array}$ & & & 0.8347 \\
\hline Well & 3 & 2 & \\
\hline Moderate & 10 & 13 & \\
\hline Poor & 15 & 13 & \\
\hline Undifferentiated & 3 & 4 & \\
\hline Invasive depth & & & 0.0009 \\
\hline $\mathrm{T} 1$ & 11 & 4 & \\
\hline $\mathrm{T} 2$ & 12 & 4 & \\
\hline $\mathrm{T} 3$ & 3 & 15 & \\
\hline $\mathrm{T} 4$ & 5 & 9 & \\
\hline FIGO stage & & & 0.0379 \\
\hline I & 5 & 3 & \\
\hline II & 15 & 7 & \\
\hline III & 8 & 19 & \\
\hline IV & 2 & 4 & \\
\hline Lymphatic metastasis & & & 0.5321 \\
\hline Yes & 16 & 14 & \\
\hline No & 15 & 18 & \\
\hline Distant metastasis & & & 0.5914 \\
\hline Yes & 4 & 4 & \\
\hline No & 22 & 33 & \\
\hline
\end{tabular}

GAS5, growth arrest-specific 5; FIGO, International Federation of Obstetrics and Gynecology. ${ }^{\mathrm{a}} \mathrm{Chi}$-squared test.

lines SKOV3, OVCAR-3, A2780, 3AO and human ovarian surface epithelium cells were detected using qRT-PCR. The results showed that GAS5 was obviously downregulated in the ovarian cancer cell lines (Fig. 2A). In addition, SKOV3 and A2780 cells were chosen for the present study. GAS5 was overexpressed in the SKOV3 and A2780 cells by pCDNA3.1-GAS5 vector transfection. The expression of GAS5 was increased 22.37- and 25.70-fold in the pCDNA3.1-GAS5transfected SKOV3 and A2780 cells compared with the vector controls, respectively (Fig. 2B). CCK-8 assay revealed that cell proliferation was significantly impaired in the pCDNA3.1GAS5-transfected SKOV3 (Fig. 2C) and A2780 (Fig. 2D) cells.

Overexpression of GSA5 suppresses ovarian cancer cell migration and invasion in vitro. To evaluate the effect of GAS5 on tumor cell migration and invasion, Transwell assay was used 
A

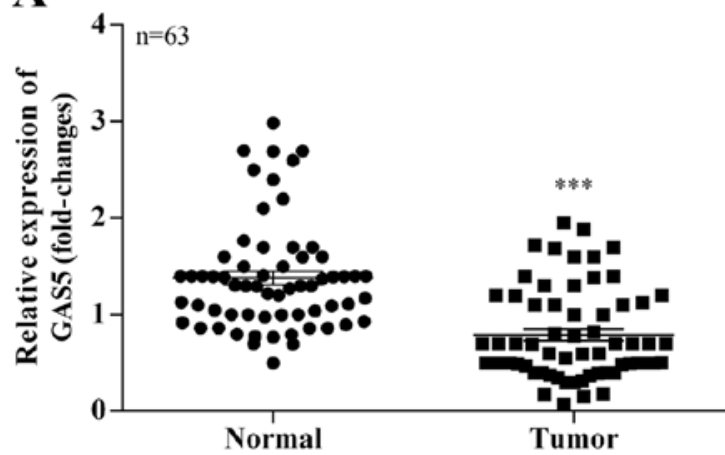

C

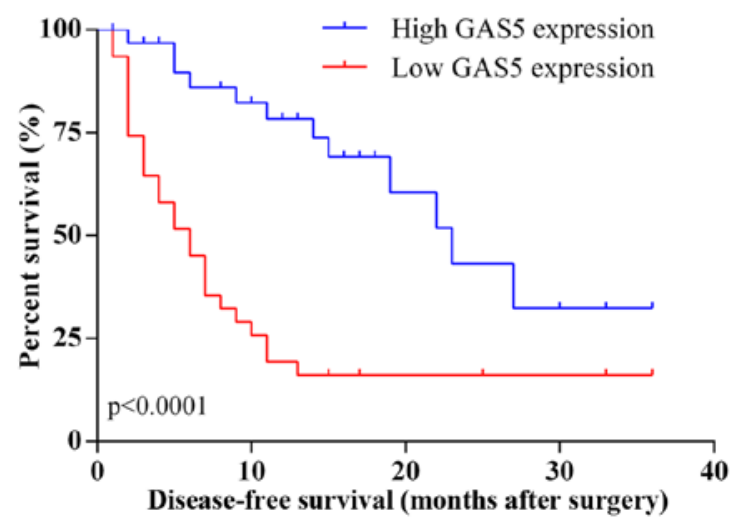

B

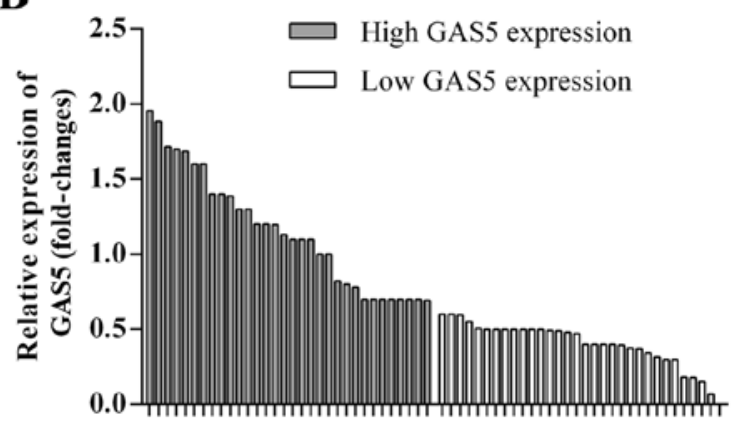

D

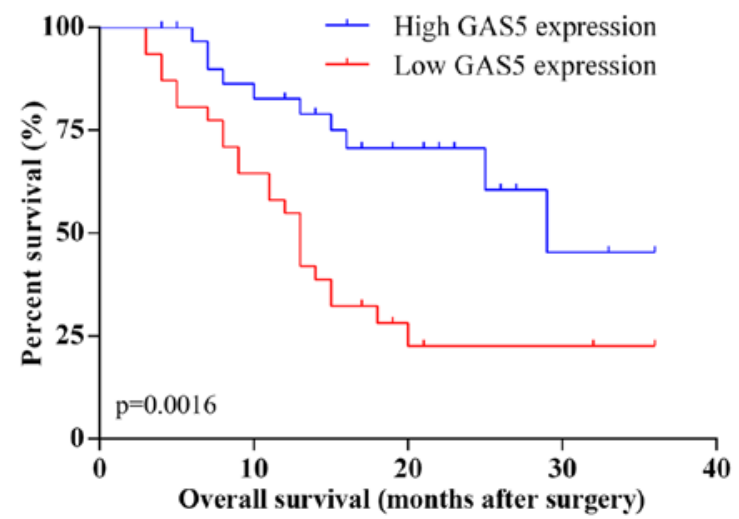

Figure 1. Expression of GAS5 in ovarian cancer tissues and its clinical significance. (A) qRT-PCR was used to detect the expression levels of GAS5 in ovarian cancer tissues $(n=63)$ and corresponding non-tumor normal tissues $(n=63)$. GAS5 was significantly downregulated in the tumor tissues; ${ }^{* * *} \mathrm{P}<0.001$. (B) According to the median ratio of GAS5 expression level (0.69) in tumor tissues, the patients were classified into two groups: high GAS5 expression group $(\mathrm{n}=32)$ and low GAS5 expression group $(\mathrm{n}=31)$. (C and D) Kaplan-Meier disease-free survival and overall survival curves according to GAS5 expression level.

A

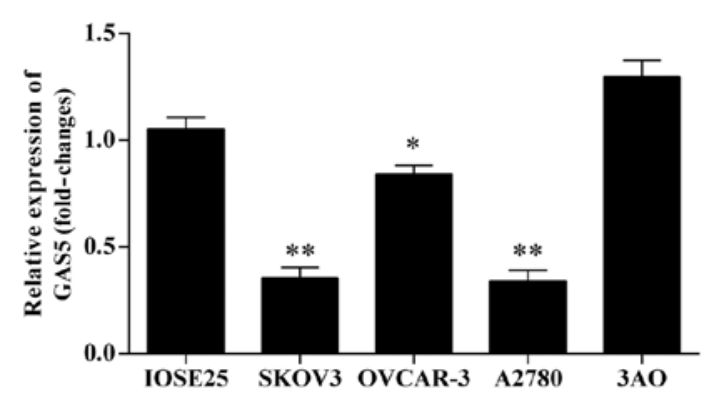

C

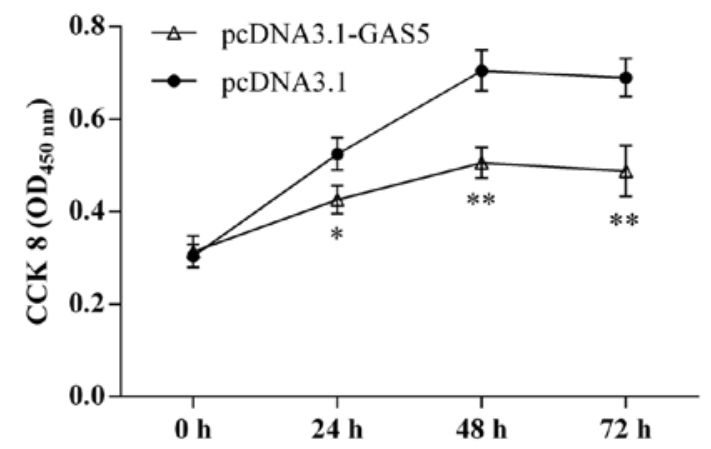

B

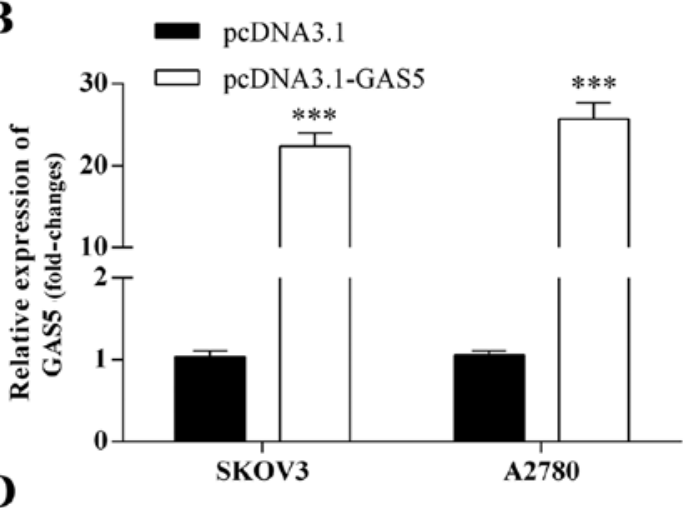

D

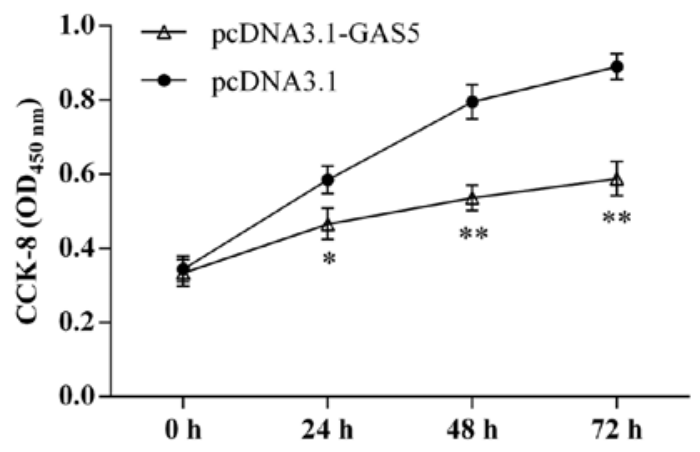

Figure 2. Overexpression of GAS5 inhibits ovarian cancer cell proliferation in vitro. (A) The expression of GAS5 in ovarian cancer cell lines (SKOV3, OVCAR-3, A2780 and 3AO) and human ovarian surface epithelial cells (IOSE25); ${ }^{*} \mathrm{P}<0.05,{ }^{* * *} \mathrm{P}<0.01$. (B) qRT-PCR analyses of the expression of GAS5 following treatment of SKOV3 and A2780 cells with pCDNA3.1-GAS5 or pcDNA3.1; ${ }^{* * *} \mathrm{P}<0.001$. (C) CCK-8 assay was performed to determine the SKOV3 cell proliferation after pCDNA3.1-GAS5 transfection; ${ }^{*} \mathrm{P}<0.05,{ }^{* *} \mathrm{P}<0.01$. (D) The proliferation of pCDNA3.1-GAS5-transfected A2780 cells was detected using CCK-8 assay; ${ }^{*} \mathrm{P}<0.05,{ }^{* *} \mathrm{P}<0.01$. 
A

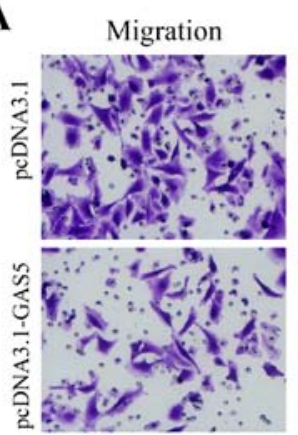

C

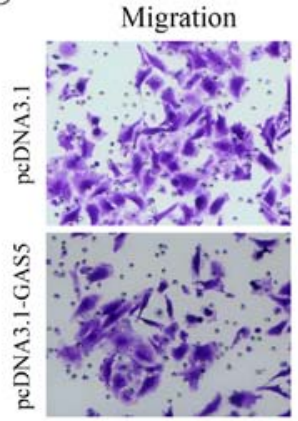

Invasion
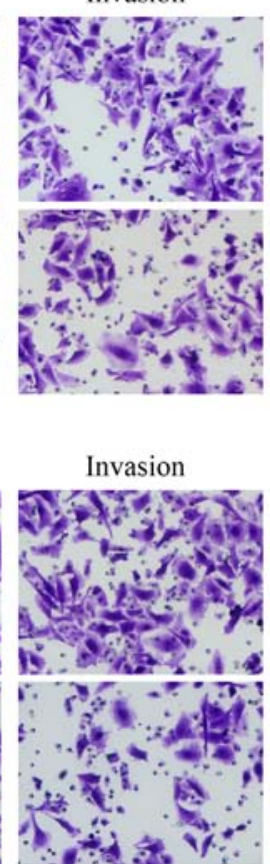

B

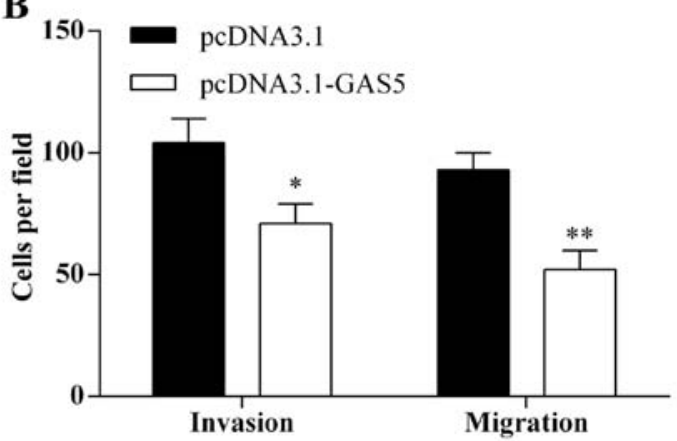

D

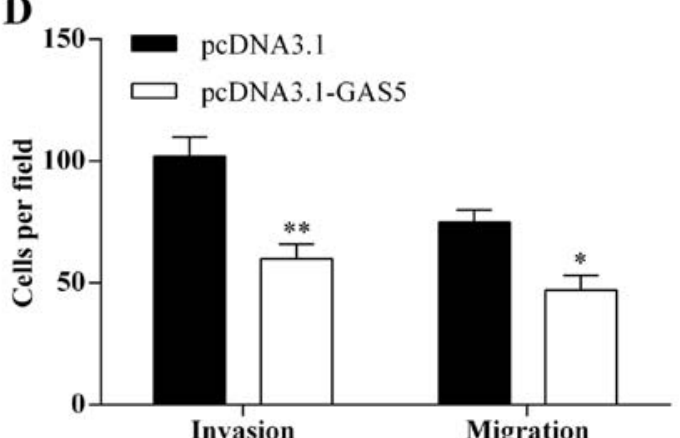

Figure 3. Overexpression of GAS5 suppresses ovarian cancer cell migration and invasion in vitro. (A) Transwell assay was used to detect SKOV3 cell migration and invasion (magnification, $\mathrm{x} 40$ ). (B) Average migration and invasion (SKOV3 cell number/field) among the different experimental groups; ${ }^{*} \mathrm{P}<0.05,{ }^{* *} \mathrm{P}<0.01$. (C) Transwell assay was used to detect A2780 cell migration and invasion (magnification, $\mathrm{x} 40$ ) (D) Average migration and invasion (A2780 cell number/field) among the different experimental groups; ${ }^{*} \mathrm{P}<0.05,{ }^{* *} \mathrm{P}<0.01$.

A

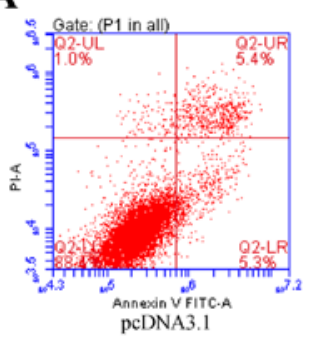

B

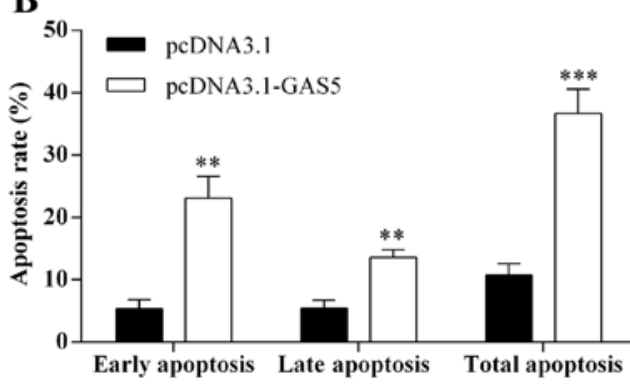

$\mathbf{E}$

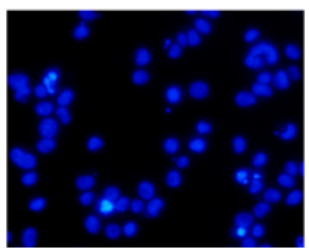

pcDNA3.1

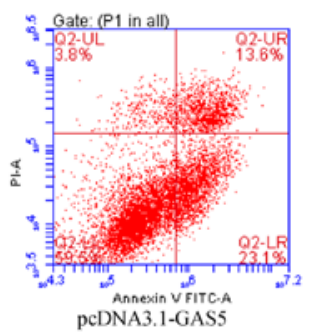

D
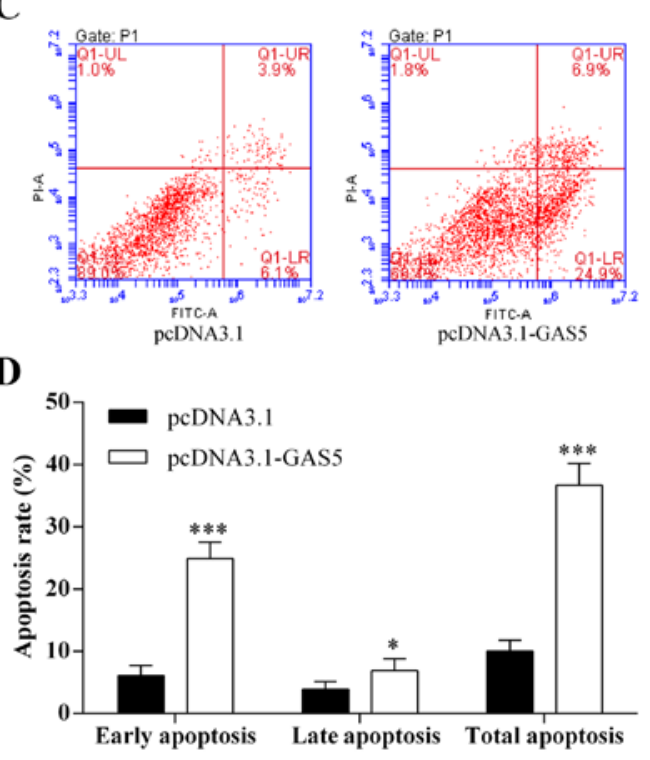

$\mathbf{F}$

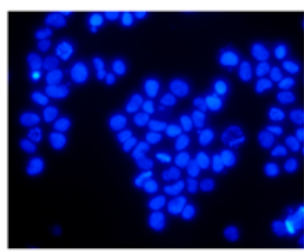

pcDNA3.1

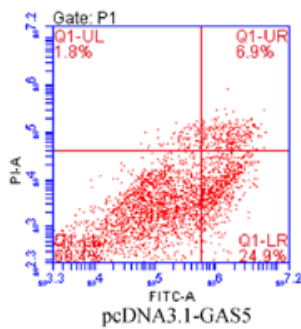

pcDNA3.1-GAS5

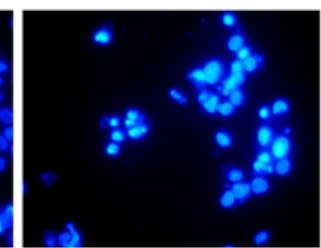

pcDNA3.1-GAS5

SKOV3

Figure 4. Overexpression of GAS5 promotes ovarian cancer cell apoptosis in vitro. Annexin V fluorescein isothiocyanate propidium iodide (FITC/PI) staining was used to monitor cell death through flow cytometry. Quadrant statistics: early apoptotic cells in lower right (LR) and late apoptotic cells in upper right (UR) quadrant; viable cells and necrotic cells shown separately in lower left (LL) and upper left (UL) quadrants. (A) Flow cytometric analysis of SKOV3 cells in the different experimental groups. (B) The percentage of early, late and total apoptotic SKOV3 cells for each group; ${ }^{* *} \mathrm{P}<0.01,{ }^{* * *} \mathrm{P}<0.001$. (C) Flow cytometric analysis of A2780 cells in the different experimental groups. (D) The percentage of early, late and total apoptotic A2780 cells for each group; $\mathrm{P}<0.05$, ${ }^{* * * *} \mathrm{P}<0.001$. (E and F) Hoechst staining assay of SKOV3 and A2780 cells with pCDNA3.1-GAS5 (or pcDNA3.1) transfection to detect apoptosis. 
$\mathbf{A}$

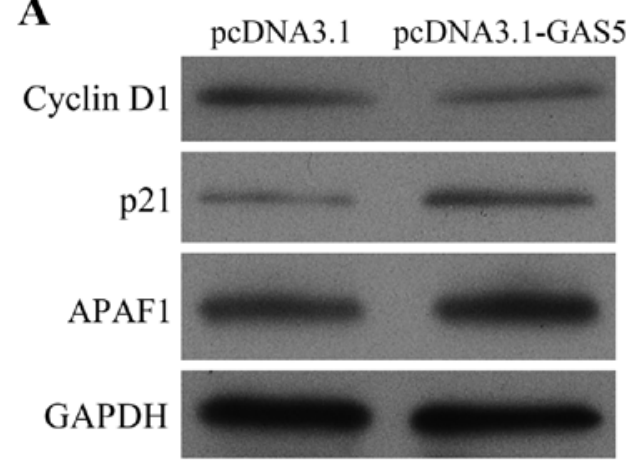

C

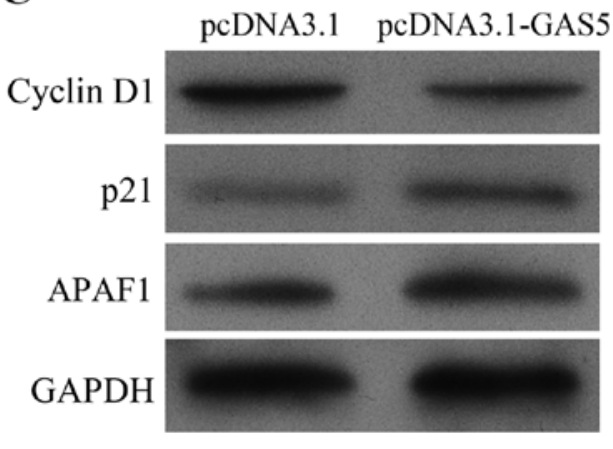

B

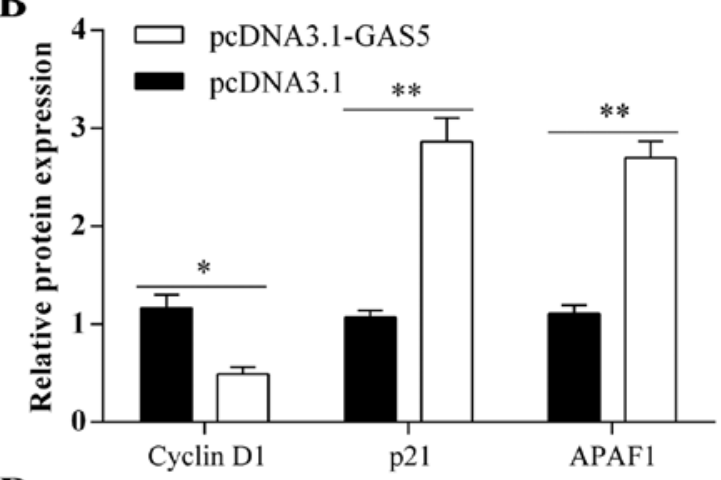

D

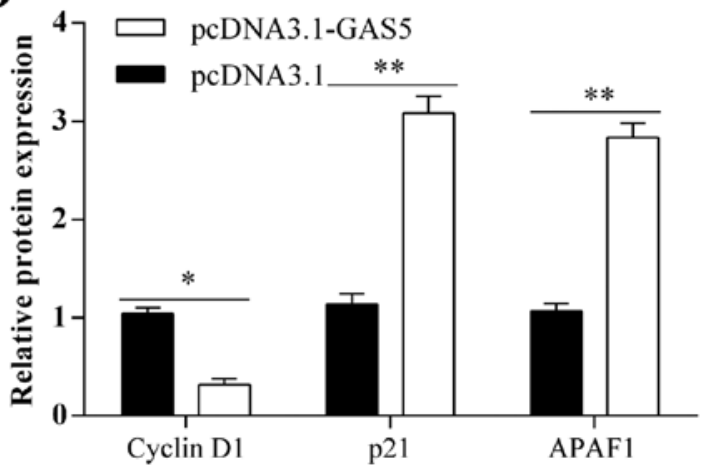

Figure 5. Overexpression of GAS5 affects cyclin D1, p21 and APAF1 protein expression. (A and B) Western blot analysis of cyclin D1, p21 and APAF1 protein expression in pCDNA3.1-GAS5 transfected SKOV3 and control cells; ${ }^{*} \mathrm{P}<0.05,{ }^{* *} \mathrm{P}<0.01$. (C and D) The protein expression levels of cyclin D1, p21 and APAF1 in pCDNA3.1-GAS5 transfected A2780 and control cells were detected using western blotting; ${ }^{*} \mathrm{P}<0.05,{ }^{* *} \mathrm{P}<0.01$.

to detect the migration and invasion abilities of the SKOV3 and A2780 cells after pcDNA3.1-GAS5 vector transfection. Overexpression of GAS5 inhibited the migration and invasion of the SKOV3 (Fig. 3A and B) and A2780 (Fig. 3C and D) cell lines.

Overexpression of GSA5 induces ovarian cancer cell apoptosis in vitro. To investigate the effect of GAS5 on cell apoptosis, the Annexin V-FITC/PI staining method was used to perform apoptosis assays. The results showed increased apoptosis rates of SKOV3 and A2780 cells following pcDNA3.1-GAS5 vector transfection compared with pcDNA3.1 vector transfection (Fig. 4). The apoptosis rates of SKOV3 cells transfected with pCDNA3.1 and pCDNA3.1-GAS5 were $10.7 \pm 1.9$ and $36.7 \pm 3.9 \%$, respectively (Fig. 4A and B). In addition, the apoptosis rates of the A2780 cells transfected with pCDNA3.1 and pCDNA3.1-GAS5 were 10.0 1.8 and $35.9 \pm 3.5 \%$, respectively (Fig. 4C and D). Moreover, Hoechst staining results showed that the number of SKOV3 and A2780 cells with condensed and fragmented nuclei following pcDNA3.1-GAS5 transfection were significantly different than the empty vector-transfected cells (Fig. 4E and F).

Cyclin D1, p21 and APAF1 are key downstream mediators of GAS5. In order to explore the underlying mechanisms involved in GAS5 overexpression-mediated suppression of cell growth, western blotting was carried out to examine the expression of potential targets after transfection with pcDNA3.1-GAS5 or pcDNA3.1 (Fig. 5). The results showed that the expression of cyclin D1 was significantly decreased in the SKOV3 (Fig. 5A and B) and A2780 (Fig. 5C and D) cells transfected with pCDNA3.1-GAS5 compared to those with pcDNA3.1. Moreover, p21 and APAF1 protein were increased in the SKOV3 (Fig. 5A and B) and A2780 (Fig. 5C and D) cells transfected with pcDNA3.1-GAS5 compared to those with pcDNA3.1. The results suggest that GAS5 may act as an oncogene by regulating cyclin D1, p21 and APAF1, and the underlying mechanism should be elucidated in further experiments.

Overexpression of GAS5 inhibits ovarian cancer cell tumorigenesis in vivo. In order to confirm the above finding that overexpression of GAS5 affects tumorigenesis, pCDNA3.1-GAS5 or pcDNA3.1 vector stably transfected SKOV3 cells were inoculated into female nude mice for 28 days following injection. The group injected with pCDNA3.1GAS5 formed substantially smaller tumors than the control group (Fig. 6A and B). Moreover, the mean tumor weight of the pCDNA3.1-GAS5 group $(0.54 \pm 0.06 \mathrm{~g})$ was markedly lower than that in the pcDNA3.1 group $(1.07 \pm 0.14 \mathrm{~g})$ at the end of the experiment (Fig. 6C). Then, the expression level of GAS5 in each neoplasm was assessed using qRT-PCR. The results showed that the expression of GAS5 in tumor tissues formed from the pCDNA3.1-GAS5 cells was significantly higher than those of tumors formed in the pcDNA3.1 group (Fig. 6D). Furthermore, western blotting was used to detect the protein expression levels of cyclin D1, p21 and APAF1 in the neoplasms. The results showed that the expression level of p21 and APAF1 protein in the neoplasms formed from the pCDNA3.1-GAS5 cells were significantly higher 
A

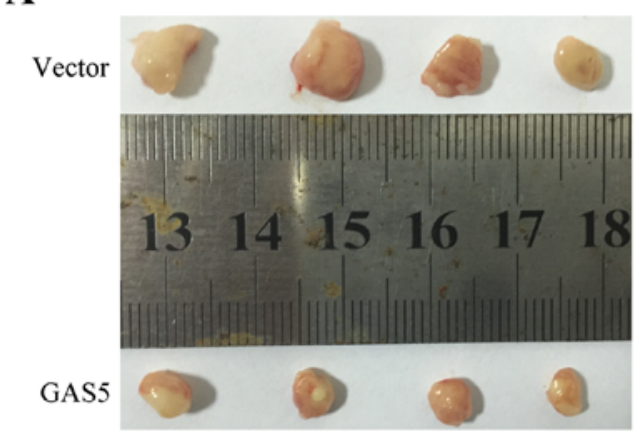

C

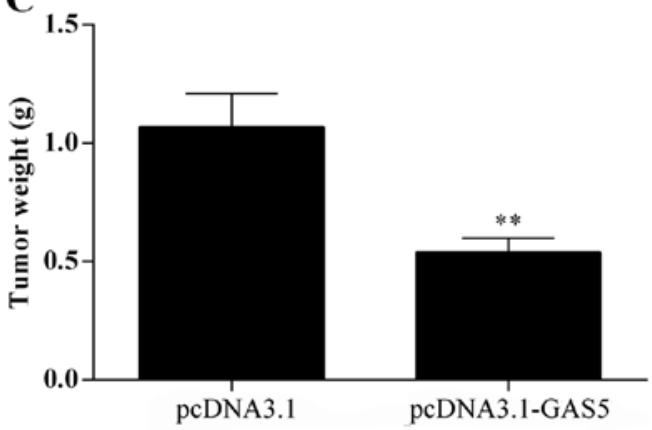

E

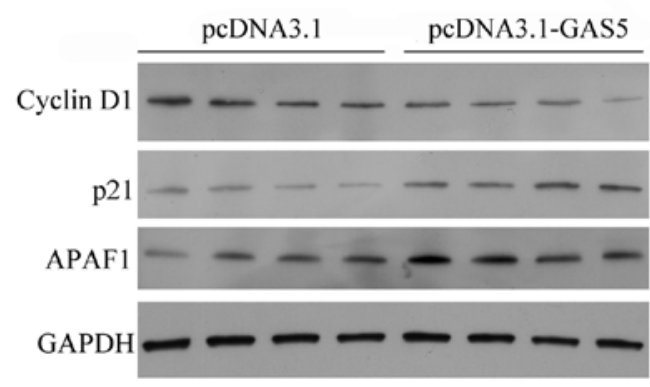

B

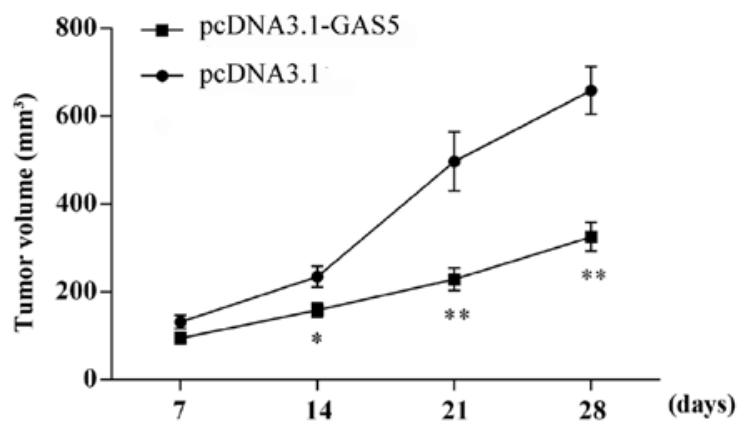

D

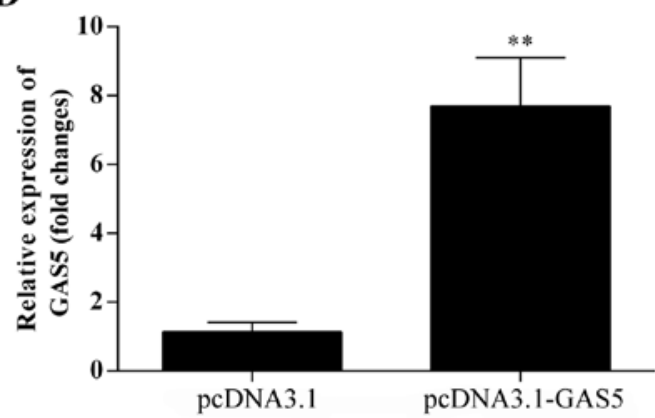

F

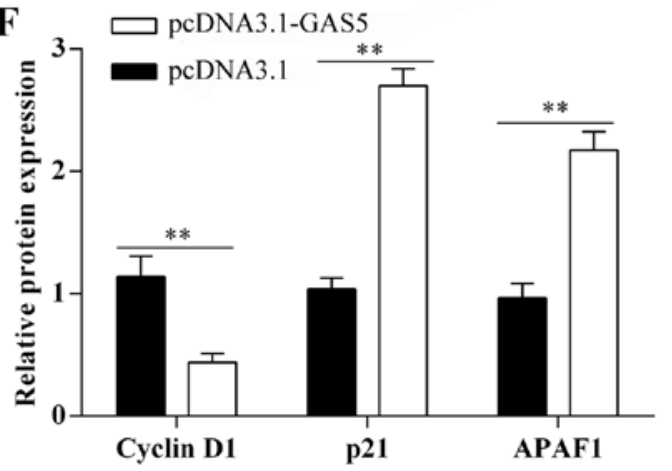

Figure 6. Overexpression of GAS5 inhibits ovarian cancer cell tumorigenesis in vivo. (A and B) The tumor volume was calculated every seven days after injection of SKOV3 cells stably transfected with pCDNA3.1-GAS5 or pcDNA3.1; ${ }^{*} \mathrm{P}<0.05,{ }^{* *} \mathrm{P}<0.01$. (C) Tumor weights are represented as means of tumor weights $\pm \mathrm{SD} ;{ }^{* *} \mathrm{P}<0.01$. (D) The expression of GAS5 in tumor tissues formed from SKOV3/pcDNA3.1-GAS5 and SKOV3/pcDNA3.1 cells were detected using qRT-PCR; ${ }^{* *} \mathrm{P}<0.01$. (E and F) Western blot analysis of cyclin D1, p21 and APAF1 protein expression in tumor tissues formed from SKOV3/pcDNA3.1-GAS5 and SKOV3/pcDNA3.1 cells. ${ }^{* *} \mathrm{P}<0.01$. Vector, pcDNA3.1; GAS5, pcDNA3.1-GAS5.

than those of the neoplasms in the pcDNA3.1 group, and the expression of cyclin D1 was significantly lower in neoplasms formed from the pCDNA3.1-GAS5 cells than the control group (Fig. 6E and F). Taken together, our results indicate that overexpression of GAS5 inhibited tumor growth and may regulate cyclin D1, p21 and APAF1 in vivo.

\section{Discussion}

In recent years, numerous studies have found that a number of lncRNAs play important roles in fundamental cellular processes, such as affecting epigenetic information and promoting cellular growth (12-17). In addition, it has been recognized that lncNRAs are involved in major pathologies, such as cancer. Dysregulation of lncRNAs results in progressive and uncontrolled tumor growth (23-25). Effective control of both cell proliferation and survival is critical to prevent oncogenesis and to treat cancer successfully. Therefore, increased understanding of the biological functions of lncRNAs may provide a missing clue toward more effective approaches for the diagnosis and treatment of ovarian cancer.

Growth arrest-specific 5 (GAS5) is a long non-coding RNA that was originally isolated in 1988 from a screen for potential tumor-suppressor genes which are expressed at high levels in growth-arrested cells (26). Previously, studies showed that GAS5 was unconventionally expressed in breast and prostate cancer, and other types of cancers (18-21). Low expression level of GAS5 is an adverse prognostic factor for survival in breast cancer and neck squamous cell carcinoma (HNSCC) $(21,27)$. In addition, overexpression of GAS5 was found to contribute to cellular growth inhibition of various cancer cell lines through regulation of the cell cycle, cell apoptosis and by promoting cell death (18-21). These data demonstrate that GAS5 is a potential tumor-suppressor. However, the role of GAS5 in the occurrence and development of ovarian cancer remains unclear.

The present study was designed to investigate the expression and prognostic significance of GAS5 in ovarian cancer 
patients. The expression level of GAS5 was retrospectively analyzed in 63 ovarian cancer patients. The results were assessed for an association with clinical features and diseasefree survival or overall survival of patients with ovarian cancer after ovariectomy. The results showed that the expression of GAS5 was significantly decreased in ovarian cancer tissues and cell lines compared with the expression noted in matched adjacent normal tissues and human ovarian surface epithelium cells. A lower expression of GAS5 was detected in tumors with larger size, deeper invasive depth and higher tumor stage. Moreover, the downregulation of GAS5 expression was associated with poor prognosis. Furthermore, overexpression of GAS5 was demonstrated to suppress ovarian cancer cell proliferation in vitro and in vivo and inhibit cell migration and invasion, and promote cell apoptosis in vitro. Taken together, our findings indicate that GAS5 acts as an anti-oncogene by regulating cell growth, invasion, migration and apoptosis, and may be useful in the diagnosis and treatment of ovarian cancer.

Although GAS5 was found to have a role as a tumor suppressor having an impact on tumorigenesis, the underlying mechanism of GAS5 in regulating gene expression is still unclear. In breast cancer, GAS5 plays a potential role as a tumor suppressor, which may be attributed to suppression of the expression of miR-21, a potential oncogene (28). Nevertheless, GAS5-mediated gene expression in ovarian pathogenesis remains unclear. In the present study, in order to explore the molecular mechanism of GAS5 that contributes to cellular growth of ovarian cancer, the potential targets of GAS5 which were responsible for cell growth and cell apoptosis were investigated. Our results confirmed that apoptosis protease activating factor 1 (APAF1), p21 as well as cyclin D1, were functional targets of GAS5 in ovarian cancer cell lines. APAF1 downregulation has been linked to decreased apoptosis and correlated with adverse prognosis in colorectal cancer (CRC) and APAFl expression is related to CRC alone or as a general mechanism in histological progression to cancer (29-31). Cyclin D1 is one of the most important proteins regulating the cell cycle, and is involved in the occurrence and development of various types of cancers. Cyclin D1 binds and activates CDK4/6, subsequently resulting in phosphorylation of tumor suppressor protein $\mathrm{Rb}$ and allows cell cycle progression through G1 into S (32). Moreover, the expression of p21 has been shown to be dysregulated in different cancer types (33). p21 prevented cell proliferation by inhibitory control of the cell cycle by direct binding to cyclins and CDKs (34). Taken together, all of our results indicate that 1ncRNA GAS5 may function as a tumor suppressor and decreased expression of GAS5 could contribute to ovarian cancer development. However, the underlying mechanism of GAS5 in regulating the expression of the above targets in ovarian cancer cells should be further studied.

In summary, we demonstrated that lncRNA GAS5 was decreased in human ovarian cancer tissues, indicating that GAS5 may play a role as a tumor-suppressor and also acts as an indicator of poor prognosis in ovarian cancer patients. A better understanding of the mechanisms of GAS5 in the evolution and progression of human ovarian cancer may lead to new diagnostic and therapeutic approaches for ovarian cancer.

\section{Acknowledgements}

The present study was approved by the Research Ethics Committee of the First Affiliated Hospital of Sun Yat-sen University.

\section{References}

1. Torre LA, Bray F, Siegel RL, Ferlay J, Lortet-Tieulent J and Jemal A: Global cancer statistics, 2012. CA Cancer J Clin 65: 87-108, 2015.

2. Breuer EK and Murph MM. The role of proteomics in the diagnosis and treatment of women's cancers: Current trends in technology and future opportunities. Int J Proteomics 2011: 373584, 2011.

3. Bertone P, Stolc V, Royce TE, Rozowsky JS, Urban AE, Zhu X, Rinn JL, Tongprasit W, Samanta M, Weissman S, et al: Global identification of human transcribed sequences with genome tiling arrays. Science 306: 2242-2246, 2004.

4. Guttman M, Amit I, Garber M, French C, Lin MF, Feldser D, Huarte M, Zuk O, Carey BW, Cassady JP, et al: Chromatin signature reveals over a thousand highly conserved large non-coding RNAs in mammals. Nature 458: 223-227, 2009.

5. Nagano $T$ and Fraser P: No-nonsense functions for long non-coding RNAs. Cell 145: 178-181, 2011.

6. Mercer TR, Dinger ME and Mattick JS: Long non-coding RNAs: Insights into functions. Nat Rev Genet 10: 155-159, 2009.

7. Cheetham SW, Gruhl F, Mattick JS and Dinger ME: Long non-coding RNAs and the genetics of cancer. Br J Cancer 108: 2419-2425, 2013.

8. Yang G, Lu X and Yuan L: LncRNA: A link between RNA and cancer. Biochim Biophys Acta 1839: 1097-1109, 2014.

9. Nie Y, Liu X, Qu S, Song E, Zou H and Gong C: Long non-coding RNA HOTAIR is an independent prognostic marker for nasopharyngeal carcinoma progression and survival. Cancer Sci 104: 458-464, 2013

10. Niinuma T, Suzuki H, Nojima M, Nosho K, Yamamoto $H$, Takamaru H, Yamamoto E, Maruyama R, Nobuoka T, Miyazaki Y, et al: Upregulation of miR-196a and HOTAIR drive malignant character in gastrointestinal stromal tumors. Cancer Res 72: 1126-1136, 2012.

11. Li J, Yang S, Su N, Wang Y, Yu J, Qiu H and He X: Erratum to: Overexpression of long non-coding RNA HOTAIR leads to chemoresistance by activating the Wnt $/ \beta$-catenin pathway in human ovarian cancer. Tumour Biol 37: 2056-2065, 2016.

12. Zhou X, Liu S, Cai G, Kong L, Zhang T, Ren Y, Wu Y, Mei M, Zhang $L$ and Wang $X$ : Long non coding RNA MALAT1 promotes tumor growth and metastasis by inducing epithelial-mesenchymal transition in oral squamous cell carcinoma. Sci Rep 5: 15972, 2015.

13. Tripathi V, Shen Z, Chakraborty A, Giri S, Freier SM, Wu X, Zhang Y, Gorospe M, Prasanth SG, Lal A, et al: Long non-coding RNA MALAT1 controls cell cycle progression by regulating the expression of oncogenic transcription factor B-MYB. PLoS Genet 9: e1003368, 2013.

14. Kotake Y, Nakagawa T, Kitagawa K, Suzuki S, Liu N, Kitagawa M and Xiong Y: Long non-coding RNA ANRIL is required for the PRC2 recruitment to and silencing of $p 15^{I N K 4 B}$ tumor suppressor gene. Oncogene 30: 1956-1962, 2011.

15. Zhu H, Li X, Song Y, Zhang P, Xiao Y and Xing Y: Long non-coding RNA ANRIL is up-regulated in bladder cancer and regulates bladder cancer cell proliferation and apoptosis through the intrinsic pathway. Biochem Biophys Res Commun 467: 223-228, 2015.

16. Su L, Han D, Wu J and Huo X: Skp2 regulates non-small cell lung cancer cell growth by Meg3 and miR-3163. Tumour Biol 37, 3925-3931, 2016.

17. Zhou Y, Zhang X and Klibanski A: MEG3 noncoding RNA: A tumor suppressor. J Mol Endocrinol 48: R45-R53, 2012.

18. Pickard MR, Mourtada-Maarabouni M and Williams GT: Long non-coding RNA GAS5 regulates apoptosis in prostate cancer cell lines. Biochim Biophys Acta 1832: 1613-1623, 2013.

19. Liu Y, Zhao J, Zhang W, Gan J, Hu C, Huang G and Zhang Y: lncRNA GAS5 enhances G1 cell cycle arrest via binding to YBX1 to regulate $\mathrm{p} 21$ expression in stomach cancer. Sci Rep 5: 10159, 2015 . 
20. Sun M, Jin FY, Xia R, Kong R, Li JH, Xu TP, Liu YW, Zhang EB, Liu XH and De W: Decreased expression of long non-coding RNA GAS5 indicates a poor prognosis and promotes cell proliferation in gastric cancer. BMC Cancer 14: 319-319, 2014.

21. Mourtada-Maarabouni M, Pickard MR, Hedge VL, Farzaneh F and Williams GT: GAS5, a non-protein-coding RNA, controls apoptosis and is downregulated in breast cancer. Oncogene 28 195-208, 2009.

22. Li NF, Broad S, Lu YJ, Yang JS, Watson R, Hagemann T, Wilbanks G, Jacobs I, Balkwill F, Dafou D, et al: Human ovarian surface epithelial cells immortalized with hTERT maintain functional pRb and p53 expression. Cell Prolif 40: 780-794, 2007.

23. Kung JT, Colognori D and Lee JT: Long non-coding RNAs: Past, present, and future. Genetics 193: 651-669, 2013.

24. Morris KV and Mattick JS: The rise of regulatory RNA. Nat Rev Genet 15: 423-437, 2014

25. Hrdlickova B, de Almeida RC, Borek $\mathrm{Z}$ and Withoff $\mathrm{S}$ : Genetic variation in the non-coding genome: Involvement of micro-RNAs and long non-coding RNAs in disease. Biochim Biophys Acta 1842: 1910-1922, 2014.

26. Schneider C, King RM and Philipson L: Genes specifically expressed at growth arrest of mammalian cells. Cell 54: 787-793, 1988.

27. Gee HE, Buffa FM, Camps C, Ramachandran A, Leek R, Taylor M, Patil M, Sheldon H, Betts G, Homer J, et al: The small-nucleolar RNAs commonly used for microRNA normalisation correlate with tumour pathology and prognosis. Br J Cancer 104: 1168-1177, 2011
28. Zhang Z, Zhu Z, Watabe K, Zhang X, Bai C, Xu M, Wu F and Mo YY: Negative regulation of lncRNA GAS5 by miR-21. Cell Death Differ 20: 1558-1568, 2013.

29. Zlobec I, Minoo P, Baker K, Haegert D, Khetani K, Tornillo L, Terracciano L, Jass JR and Lugli A: Loss of APAF-1 expression is associated with tumour progression and adverse prognosis in colorectal cancer. Eur J Cancer 43: 1101-1107, 2007.

30. Luo X, Burwinkel B, Tao S and Brenner H: MicroRNA signatures: Novel biomarker for colorectal cancer? Cancer Epidemiol Biomarkers Prev 20: 1272-1286, 2011.

31. Paik SS, Jang KS, Song YS, Jang SH, Min KW, Han HX, Na W, Lee KH, Choi D and Jang SJ: Reduced expression of Apaf-1 in colorectal adenocarcinoma correlates with tumor progression and aggressive phenotype. Ann Surg Oncol 14: 3453-3459, 2007.

32. Fu M, Wang C, Li Z, Sakamaki T and Pestell RG: Minireview: Cyclin D1: normal and abnormal functions. Endocrinology 145: 5439-5447, 2004.

33. Abbas T and Dutta A: p21 in cancer: Intricate networks and multiple activities. Nat Rev Cancer 9: 400-414, 2009.

34. Chen J, Saha P, Kornbluth S, Dynlacht BD and Dutta A: Cyclin-binding motifs are essential for the function of $\mathrm{p} 21^{\text {CIPI }}$. Mol Cell Biol 16: 4673-4682, 1996. 